\title{
Time-lapse angiography of the ocular fundus: a new video-angiography
}

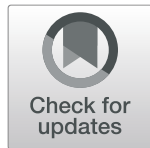

Chufeng $\mathrm{Gu}^{1 \dagger}$, Jili Chen ${ }^{2 \dagger}$, Tong Su${ }^{1 \dagger}$ and Qinghua Qiur, ${ }^{1,3^{*}}$

\begin{abstract}
Background: Ocular fundus angiography is an indispensable component of the tests utilized for fundus diseases. Dynamic angiography results can provide additional information; however, many difficulties remain. In this study, we introduce a modified method, time-lapse angiography (TLA), to dynamically present imaging results.

Methods: TLA, combining time-lapse photography and fundus angiography (using Heidelberg retina angiography II, Germany), includes pre-photographing and post- photosynthesis and ultimately produces a video that is approximately $15 \mathrm{~s}$ in length.

Results: Four typical videos in the article showed the characteristics of TLA, including a short and rapid but continuous and integral presentation, highly valid information, high definition, etc.

Conclusions: TLA is beneficial for the diagnosis of diseases and the assessment of progression and is convenient for peer communication, patient interpretation, and student education. The application of time-lapse photography in ocular fundus angiography is a monumental and innovative attempt.
\end{abstract}

Keywords: Time-lapse photography, Ocular fundus angiography, Three-dimensional visualization, Dynamic report

\section{Background}

Fluorescein fundus angiography (FFA) and indocyanine green angiography (ICGA) are indispensable components of the tests utilized for fundus diseases. Conventionally, imaging results are recorded by an ophthalmic medical technician, who selects a few typical images and uses a paper-based imaging report to present the results. This downgrades the dynamic changes in eye-ground vessels into isolated and static ones. Additionally, the amount of information presented to doctors is greatly reduced. Thus, determining how to record the complete angiography process is highly important.

One of the best devices to use is a Heidelberg retina angiography II (HRA II, Heidelberg Engineering, Heidelberg, Germany), which can record short, one-minute

\footnotetext{
* Correspondence: qinghuaqiu@163.com

${ }^{+}$Chufeng Gu, Jili Chen and Tong Su contributed equally to this work. 'Department of Ophthalmology, Shanghai General Hospital, Shanghai Jiao Tong University School of Medicine; Shanghai Key Laboratory of Ocular Fundus Diseases; Shanghai Engineering Center for Visual Science and Photomedicine, Shanghai 200080, People's Republic of China

${ }^{3}$ Department of Ophthalmology, Shigatse People's Hospital, Xizang 857000, People's Republic of China

Full list of author information is available at the end of the article
}

videos, but the imaging process takes more than $10 \mathrm{~min}$. In addition, being able to video-record the entire process, store data and deliver traditional videos is not easy when dealing with high-volume data, which contains too much invalid information. Consequently, if we could invent new technology to solve these problems, we could improve the process.

Time-lapse photography is a recording technique that captures images at a much lower frequency than the frequency that is applied to view the sequence. As a result, when played at a normal speed, time seems to move faster, which creates the lapse. Currently, there are a variety of applications for this technology in the medical field, such as for the diagnosis of human preimplantation embryo viability [1] and the quantification of bone resorption and formation [2]. In ophthalmology, time-lapse photography has been used to illustrate the development of idiopathic macular holes and the effects of surgical treatment [3]. However, applying time-lapse photography in ocular fundus angiography to assist in the diagnosis of ophthalmic diseases has not been reported.

We wish to introduce a modified method, time-lapse angiography (TLA), that combines time-lapse 
photography and fundus angiography (using HRA II) to dynamically present imaging results.

\section{Methods}

TLA includes pre-photographing and post-photosynthesis. First, we took 3-5 fundus photographs before injecting fluorescein. Then, we used time-lapse photography to take at least 20 images per minute from the beginning of the angiography process, containing the pre-arterial phase, retinal arterial stage, arterial-venous stage, venous stage, and late-venous stage. In principle, we needed more than 300 images. After removing the blurry images caused by moving and blinking of the eyes, we used the following software to process and synthesize the remaining photos: LRTimelapse (Germany), Adobe Photoshop Lightroom (Adobe Systems Incorporated, USA), and Adobe After Effects (Adobe Systems Incorporated, USA).

\section{Results}

In the $12 \mathrm{~s}$ stereoscopic video (Video S1, Additional file 1 ), we observed that fluorescein leaked from the retinal vessels to the outside and eventually into the vitreous cavity in the late staining stage. This suggested that the lesion had already affected the vitreous cavity. However, this phenomenon may be confused with retinal vascular leakage in a planar and static report (Fig. 1).

Video S2 (Additional file 2), approximately $17 \mathrm{~s}$ in length, specifically and representatively shows the entire fluorescence filling process of cystoid macular edema: the blurry leakage sites of the microvessels around the fovea became increasingly larger in the late-venous stage, fusing with each other and forming petal-like or radial appearance spots around the fovea. This is especially suited for teaching. Furthermore, we discovered that a flocculent shadow existed in front of the retina throughout these stages, which represents the performance of the vitreous opacity; however, this finding is likely to be considered hypofluorescence in a planar, paper-based report (Fig. 2).

Video S3 (20 s, Additional file 3) and Video S4 (19 s, Additional file 4) are the results of FFA and ICGA, respectively, for retinal pigment epithelium detachment similar to the evolution of stars and the eternity of black-holes - which fully embodies the artistic beauty
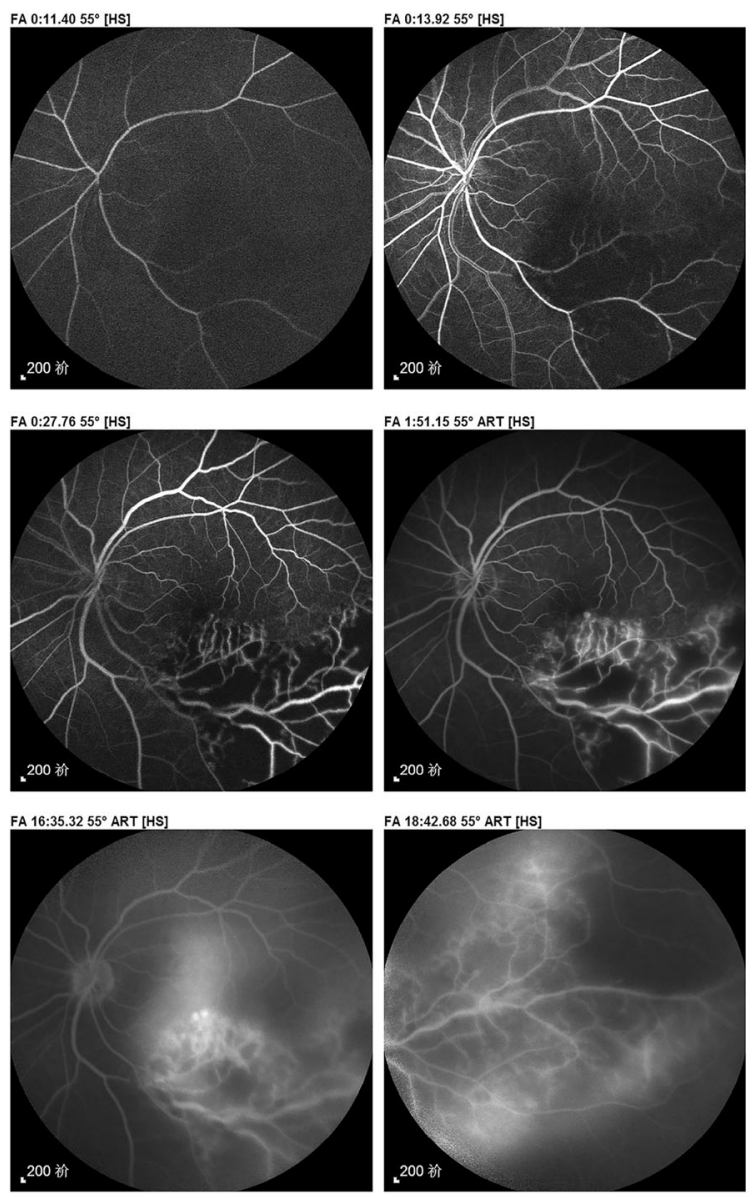
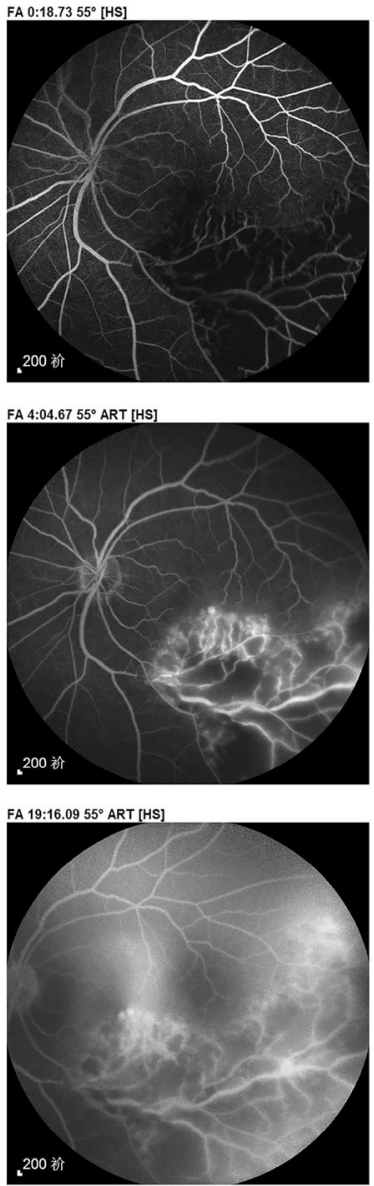

Fig. 1 The paper-based imaging report corresponding to Video S1 

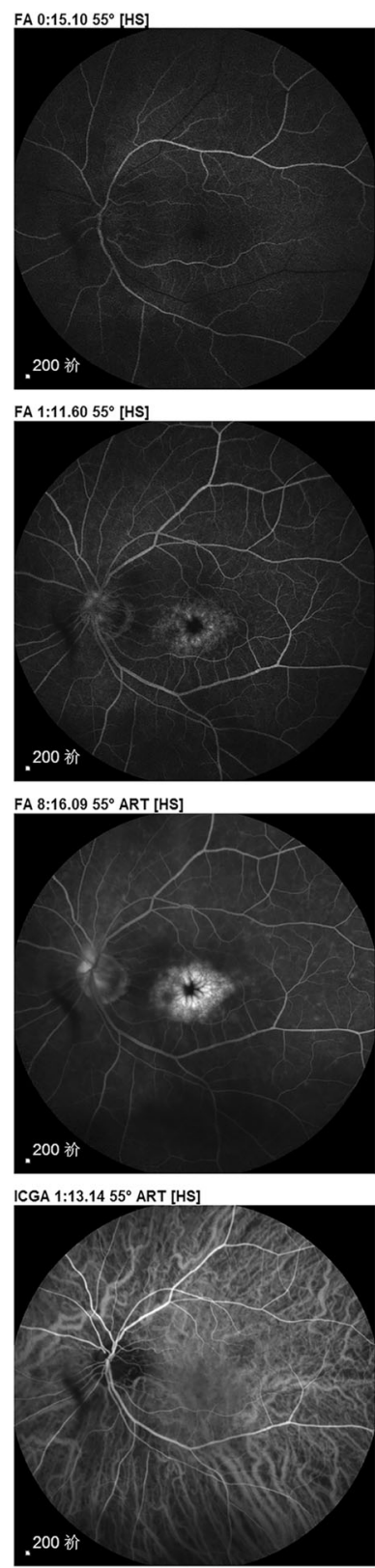

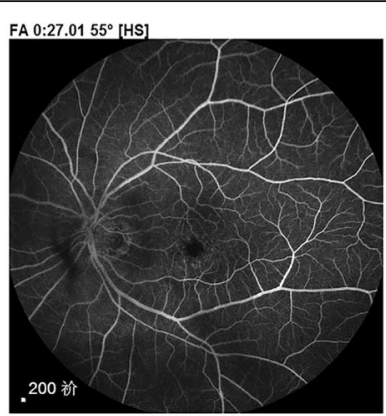

FA 1:13.67 55० ART [HS]

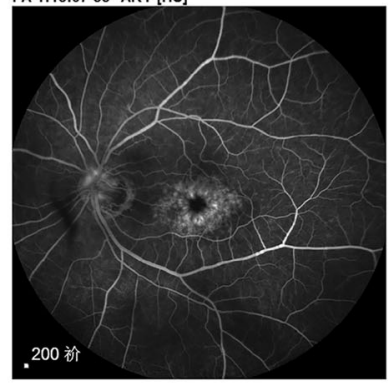

FA 18:38.98 55० ART [HS]

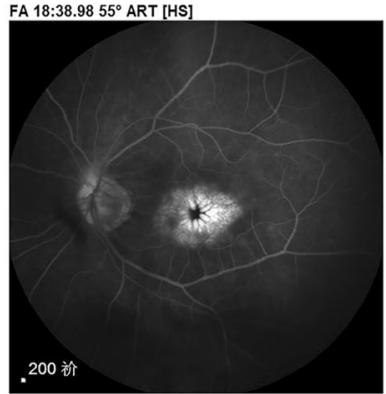

ICGA 5:19.01 55० ART [HS]

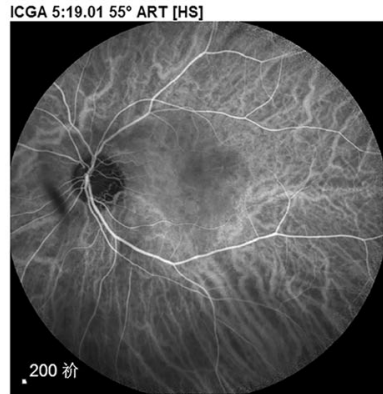

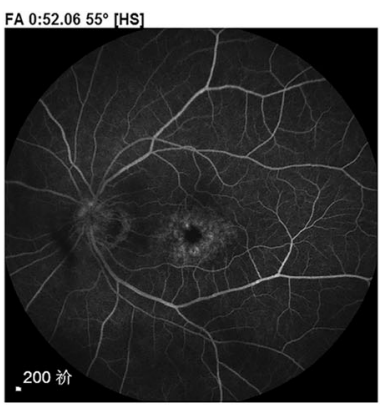

FA 1:46.50 55० ART [HS]

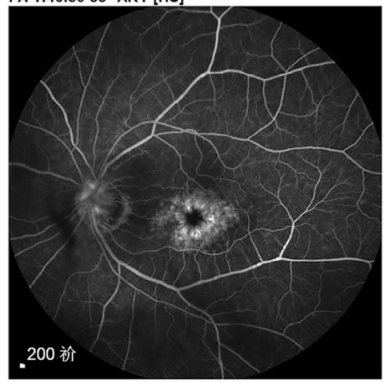

ICGA 0:19.60 $55^{\circ}[\mathrm{HS}]$

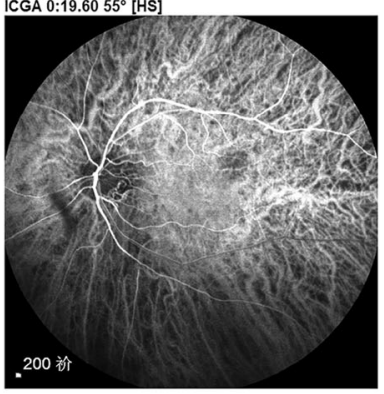

ICGA 31:30.90 55० ART [HS]

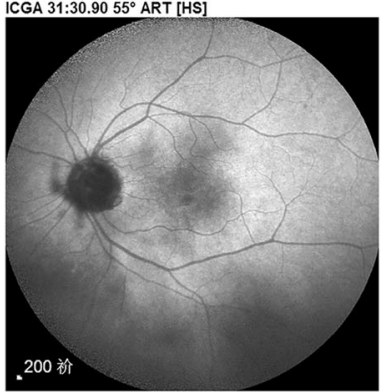

Fig. 2 The paper-based imaging report corresponding to Video S2

of video-angiography. The corresponding paper-based imaging report was shown in Fig. 3.

\section{Discussion}

Just as films have developed from 2D to 3D to Imax, and as the form of artistic expression has changed from a flat surface into a three-dimensional one, the development of ocular fundus angiography reports from static and planar to dynamic and stereoscopic is an inevitable trend. This study introduces TLA to stereoscopically present imaging results. Although TLA only combines two established methods, this innovation is beneficial for the diagnosis of diseases and the assessment of progression. TLA reconnects static images and turns fundus angiography into a real dynamic check, which brings the three-dimensional aspect of imaging results to life (Videos S1 and S2). Moreover, TLA solves the deficiency of traditional videos with low resolution and high-volume data (within $20 \mathrm{~s}$ ). This new video-angiography maintains the advantages of high definition in the paperbased reports and massive amounts of valid information in the conventional video. We can choose the 

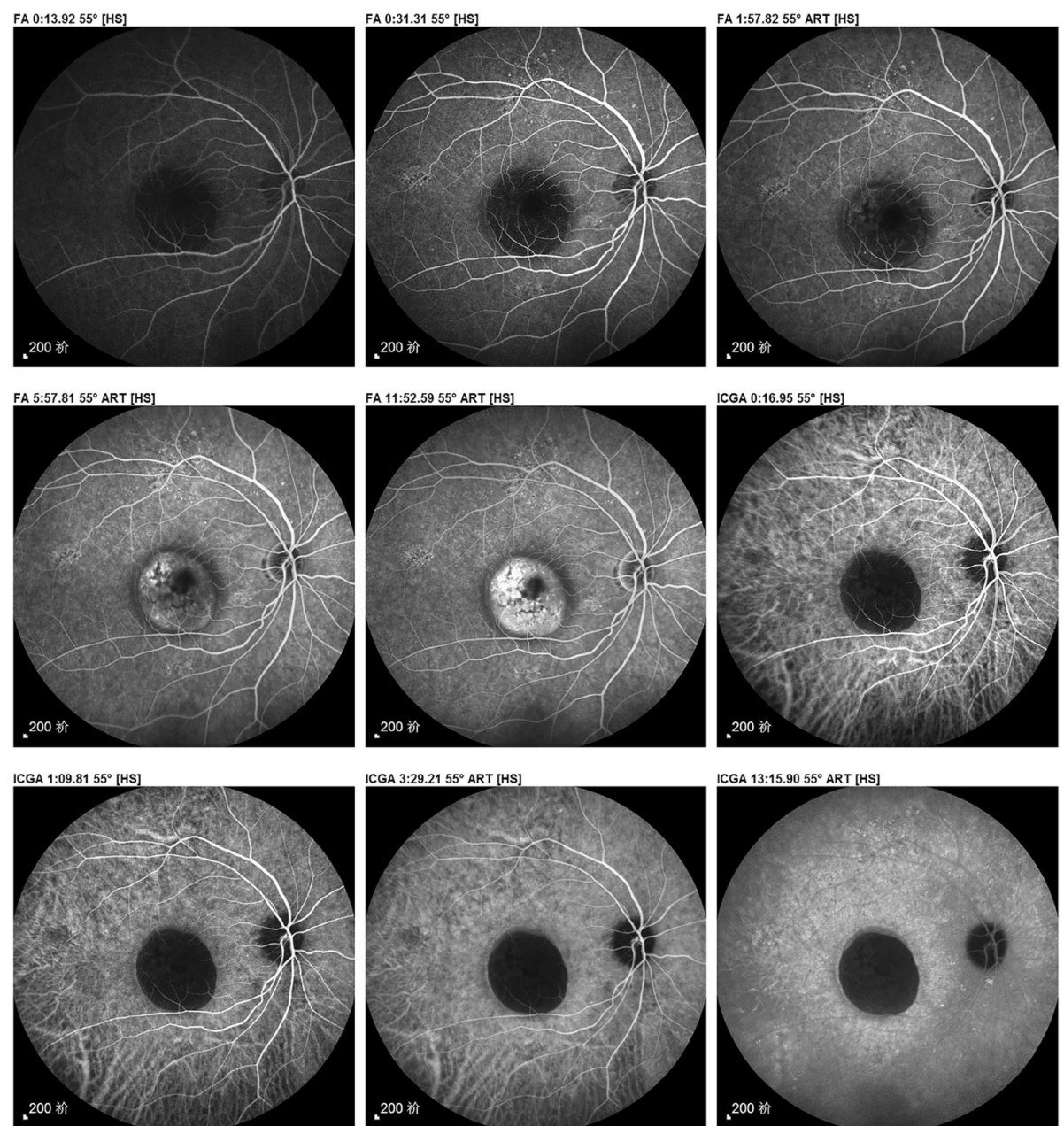

Fig. 3 The paper-based imaging report corresponding to Videos S3 and S4

appropriate window of time to observe dynamic changes in specific parts of fundus vessels, especially for the vasa vasorum and new vessels. In addition to facilitating a doctor's diagnosis, this dynamic report is convenient for peer communication, patient interpretation, and student education. Furthermore, this technique is applicable to other areas of medical angiography and may bring new inspiration to the development of imaging technology. However, further studies with a large sample and quantitatively statistical analysis are necessary to get more objective results.

\section{Conclusions}

TLA is beneficial for the diagnosis of diseases and the assessment of progression, and it is convenient for peer communication, patient interpretation, and student education. The application of time-lapse photography in ocular fundus angiography is a monumental and innovative attempt.

\section{Supplementary information}

Supplementary information accompanies this paper at https://doi.org/10. 1186/s12880-019-0398-1.

Additional file 1. Video S1: TLA of fundus diseases involving the vitreous cavity.

Additional file 2. Video S2: TLA of cystoid macular edema.

Additional file 3. Video S3: TLA of FFA for retinal pigment epithelium detachment.

Additional file 4. Video S4: TLA of ICGA for retinal pigment epithelium detachment

\section{Abbreviations}

FFA: Fluorescein fundus angiography; HRA II: Heidelberg retina angiography II; ICGA: Indocyanine green angiography; TLA: Time-lapse angiography

\section{Acknowledgements}

Not applicable.

Authors' contributions

CG, JC and TS performed the experiments and contributed in writing the manuscript. QQ revised manuscript. All authors read and approved the final manuscript. 


\section{Funding}

The design of the study was supported by the Advanced and Appropriate Technology Promotion Project of Shanghai Health Commission (grant number: 2019SY012); the collection, analysis, and interpretation of data were supported by the Natural Science Foundation of Shanghai and Jingan District (grant number: 201740001; grant number: 2018MS12); writing the manuscript was supported by the National Natural Science Foundation of China (grant number: 81970811)

\section{Availability of data and materials}

The datasets used and analysed during the current study are available from the corresponding author on reasonable request.

\section{Ethics approval and consent to participate}

This protocol was approved by the Ethics Committee of Shanghai General Hospital, Shanghai Jiao Tong University School of Medicine. All patients were informed according to the World Medical Association Declaration of Helsinki, and the written informed consent was obtained from all participants.

\section{Consent for publication}

Not applicable.

\section{Competing interests}

The authors declare that they have no competing interests.

\section{Author details}

'Department of Ophthalmology, Shanghai General Hospital, Shanghai Jiao Tong University School of Medicine; Shanghai Key Laboratory of Ocular Fundus Diseases; Shanghai Engineering Center for Visual Science and Photomedicine, Shanghai 200080, People's Republic of China. ${ }^{2}$ Department of Ophthalmology, Shibei Hospital of Jing'an District, Shanghai 200040, People's Republic of China. ${ }^{3}$ Department of Ophthalmology, Shigatse People's Hospital, Xizang 857000, People's Republic of China.

Received: 29 September 2019 Accepted: 3 December 2019 Published online: 19 December 2019

\section{References}

1. Gardner DK, Meseguer M, Rubio C, Treff NR. Diagnosis of human preimplantation embryo viability. Hum Reprod Update. 2015;21(6):727-47.

2. Christen P, Muller R. In vivo visualisation and quantification of bone Resorption and bone formation from time-lapse imaging. Curr Osteoporos Rep. 2017;15(4):311-7.

3. Gentile RC, Landa G, Pons ME, Eliott D, Rosen RB. Macular hole formation, progression, and surgical repair: case series of serial optical coherence tomography and time lapse morphing video study. BMC Ophthalmol. 2010; 10:24

\section{Publisher's Note}

Springer Nature remains neutral with regard to jurisdictional claims in published maps and institutional affiliations. 